\title{
PARALLEL STUDY OF HEMORHEOLOGICAL, INFLAMMATION AND ANEMIA PARAMETERS BEFORE, DURING AND AFTER SURGERY IN PATIENTS WITH STOMACH CANCER
}

\author{
Ekaterene Labadze ${ }^{1}$, Aleksande Tsalugelashvili ${ }^{4}$, Maya Mantskava ${ }^{2,3}$, \\ Nana Momtselidze ${ }^{2}$, Nugzar Narsia ${ }^{1}$ \\ ${ }^{1}$ Tbilisi State Medicial University, Tbilisi, Georgia \\ ${ }^{2}$ Beritashvili Experimental Center of Biomedicine, Tbilisi, Georgia \\ ${ }^{3}$ Perm Technique University, Tbilisi, Georgia \\ ${ }^{4}$ New vision University Hospital
}

DOI: https://doi.org/10.31435/rsglobal_sr/31072019/6609

\section{ARTICLE INFO}

Received 28 May 2019

Accepted 15 July 2019

Published 31 July 2019

\section{KEYWORDS}

stomach cancer, hemorheology, inflammation, anemia,

local blood flow.

\begin{abstract}
The aim of our work was to study the biomechanical markers of blood flow in patients with stomach cancer. Therefore, we paid attention to the parameters of hemorheology, rheology, inflammation and anemia in the group of patients with stomach cancer and in the control group; with the help of a new surgical technique, we simultaneously examined the flow parameters in the central blood and locally at the site of tumor growth during the operation. As a result of the matrix analysis and statistical data processing, it turned out that in the group of patients the average values of hemorheological parameters went beyond the limits of the clinical norm and significantly differed from the parameters in the control group. Moreover, the parameters of inflammation and anemia also differed in the group of patients from the values of the control group, but with a little difference. Consequently, the parameters of hemorheology, inflammation, and the parameters that determine the presence of anemia are variable in the local circulation as compared to the central one, mainly due to the hemorheological component.
\end{abstract}

Citation: Ekaterene Labadze, Aleksande Tsalugelashvili, Maya Mantskava, Nana Momtselidze, Nugzar Narsia. (2019) Parallel Study of Hemorheological, Inflammation and Anemia Parameters Before, During and after Surgery in Patients with Stomach Cancer. Science Review. 6(23). doi: 10.31435/rsglobal_sr/31072019/6609

Copyright: (C) 2019 Ekaterene Labadze, Aleksande Tsalugelashvili, Maya Mantskava, Nana Momtselidze, Nugzar Narsia. This is an open-access article distributed under the terms of the Creative Commons Attribution License (CC BY). The use, distribution or reproduction in other forums is permitted, provided the original author(s) or licensor are credited and that the original publication in this journal is cited, in accordance with accepted academic practice. No use, distribution or reproduction is permitted which does not comply with these terms.

Introduction. Stomach cancer is a cancer, which develops from the lining of the stomach. There are early symptoms (heartburn, upper abdominal pain, nausea and loss of appetite) and later symptoms (weight loss, yellowing of the skin and whites of the eyes, vomiting, difficulty swallowing and blood in the stool). The cancer may spread from the stomach to other parts of the body [1]. Most of the time, stomach cancer develops in stages over years [2]. Diagnosis is usually by biopsy done during endoscopy [3]. Medical imaging to determine if the disease has spread to other parts of the body [3] follows this. Treatments may include some combination of surgery, chemotherapy, radiation therapy and targeted therapy [3,4]. If treated late, palliative care may be advised [2]. Globally, stomach cancer is the fifth leading cause of cancer and the third leading cause of death from cancer, making up $7 \%$ of cases and $9 \%$ of deaths [5]. These data indicate the relevance of the topic. We looked at the problems in the new perspective. The aim of our work was to study the biomechanical markers of blood flow in patients with stomach cancer. Therefore, we paid attention to the parameters 
of hemorheology, rheology, inflammation and anemia in the group of patients with stomach cancer and in the control group.

Materials and methods. We examined 3 men, with stomach cancer. The diagnosis of stomach cancer was made by studying the morphology by a microscope. All patients were prescribed surgery in accordance with the medical therapeutic standard. Their average age was $62 \pm 2.08$ years. The duration of the disease could not be identified, since the inclusion of patients in our group was carried out immediately after diagnosis. From the moment of diagnosing cancer prior to the operation, standard studies were carried out in patients, approved by the Ministry of Labor, Health and Social Protection of Georgia. All patients had positive rhesus and the first blood group. We investigated the parameters determining the rheological state of the blood, the presence of anemia and inflammation as biomechanical determinants of the blood flow. Specifically erythrocytes aggregation index (EAI), erythrocytes deformation index (EDI), blood plasma viscosity (IBP) and HCT, ESR, WBC, FB, CRP, HGB, RBC, MCV, MCH. We investigated these parameters: 1) in patients before the surgery 2 hours prior to the premedication in $\mathrm{v}$. cubital is; 2) during the operation in - a) the local blood flow of the patient in v. Spleen Potrae and b) in the central circulation in v.Cubitalis of a patient. We investigated in analogical parameters in the simples of blood from v.Cubitalis in control group. Blood from v.Cubitalis blood was collected in accordance with laboratory standards. We proposed an innovative method for studying the fluidity of blood directly at the tumor growth point, in order to study the local blood flow during surgery. In this regard, we took blood locally from v. Spleen Portae. Firstly, we conducted a revision of the entrance to the spleen and nearby lymph nodes, mobilized gastro-spleen yogi, founding an artery and vein at the same time. The vein was taken into two ligatures and blood was taken respectively. For control, we selected three healthy men of respective age and weight, with the first positive blood group, in which we conducted similar studies that were conducted in our patients before surgery. We took the informational consent in both of patients and persons in control group in accordance with the International Standards [6], the form of which was specially prepared by our organizations and approved by the Ethical Committee of the I. Beritashvili Center for Experimental Studies of Biomedicine. We used the optimized texture analysis method "Tas-plas" [7] Leits, Germany) to determine the rheological parameters. The statistical analysis was carried out by the "Origin 8.1 for IoS" statistical processing program (USA).

Results. Our studies showed that in the group of patients, the average values of hemorheological parameters went beyond the limits of the clinical norm and significantly differed from the parameters in the control group. The parameters of inflammation and anemia also differed in the group of patients from the values in the control group, but with a lesser percentage degree, which was unreliable as well. According to our data, the parameters of hemorheology, inflammation, and determining the presence of anemia are variable in the local circulation as compared with the central one, mainly due to the hemorheological component. The data of all parameters are in Table \#1.

Table 1. Parameters of hemorheology, inflammation, anemia in patients group and in sontrol group. ( $\mathrm{M} \pm \mathrm{m})$.

\begin{tabular}{|c|c|c|c|c|c|c|c|c|c|c|c|c|}
\hline & \multicolumn{4}{|c|}{ Hemorheologycal Parameters } & \multicolumn{4}{|c|}{ Inflammation Parameters } & \multicolumn{4}{|c|}{ Parameters of Anemia } \\
\hline & EAI,\% & EDI,\% & IBV, sP & Hct, $\%$ & $\begin{array}{l}\text { ESR, } \\
\%\end{array}$ & $\begin{array}{l}\text { WBC } \\
10^{9} / 1 \\
\end{array}$ & $\begin{array}{l}\mathrm{CRP} \\
\mathrm{mg} / \mathrm{l}\end{array}$ & $\mathrm{FBg} / \mathrm{l}$ & $\mathrm{HGB}, \mathrm{g} / \mathrm{l}$ & $\begin{array}{l}\text { RBC, } \\
10^{12}\end{array}$ & $\mathrm{MCV}$ & $\begin{array}{l}\mathrm{MCH}, \\
\mathrm{g} / \mathrm{l} / \mathrm{hgb}\end{array}$ \\
\hline 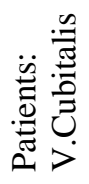 & $\begin{array}{l}+ \\
\text { ते } \\
\text { ले }\end{array}$ & $\begin{array}{l}n \\
0 \\
0 \\
+ \\
i \\
i \\
\text { in }\end{array}$ & 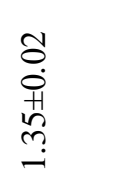 & $\begin{array}{l}\stackrel{+}{\oplus} \\
\stackrel{\oplus}{f}\end{array}$ & $\begin{array}{l}\text { î } \\
\text { 茪 } \\
\text { in }\end{array}$ & 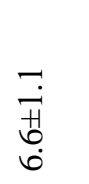 & 年 & $\begin{array}{l}\tilde{H} \\
\text { 1 } \\
\text { in }\end{array}$ & $\stackrel{n}{\stackrel{n}{1}}$ & $\begin{array}{l}\dot{0} \\
\stackrel{+}{+} \\
\infty \\
\dot{+}\end{array}$ & $\begin{array}{l}0 \\
\text { i } \\
\text { n } \\
\infty \\
\infty\end{array}$ & 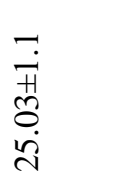 \\
\hline 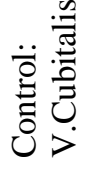 & $\begin{array}{l}n \\
\text { in } \\
\text { i } \\
\infty\end{array}$ & 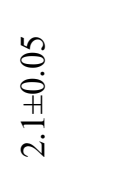 & 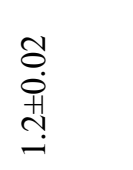 & 势 & $\frac{0}{\dot{H}}$ & $\begin{array}{l}\stackrel{N}{+} \\
\text { m } \\
\stackrel{+}{+}\end{array}$ & $\begin{array}{l}n \\
\text { On } \\
\forall\end{array}$ & 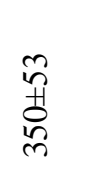 & $\begin{array}{l}\frac{0}{H} \\
\text { 苔 }\end{array}$ & 等 & $\frac{N}{\not}$ & $\begin{array}{l}\text { तु } \\
\text { Hु } \\
\text { ते }\end{array}$ \\
\hline
\end{tabular}

Discussions. Our studies showed that before the operation in blood taken from v.cubitalis, the average values of rheological parameters, parameters of inflammation and anemia in patients with cancer differed compared with the corresponding parameters of the control group, however some of them ranged within the existing clinical and therapeutic standards. We found that the average values differed from each other in cancer patients during surgery, in blood samples taken from v.cubitalis and 
v.spleen potrae. Moreover, average values of the studied parameters of blood samples taken from v.cubitalis corresponded to the average values of similar parameters in blood samples before the operation, which may mean that the studied factors are resistant to anesthesia and other stressors that affect the body in the operation. This speaks in favor to the tactics and algorithm of the surgical intervention itself for stomach cancer. However, our main goal was to study the biomechanical changes in the fluidity of central blood and locally in the area of neoplasms. In order to describe mathematically the biomechanical changes, the clinical data of which we presented in the "Results" section of this article, we reviewed the data area. This is the 12-factor set (EAI, EDI, IBV, HCT, ESR, $\mathrm{WBC}, \mathrm{FB}, \mathrm{CRP}, \mathrm{HGB}, \mathrm{RBC}, \mathrm{MCV}, \mathrm{MCH}$ ) for 2 degrees of freedom (blood from v.Cubitalis and blood from v. Spleen portae) in the case of n patients (3 patients). We will create matrixes [8], the elements of which will correspond to the difference between the measured parameters in the local and central blood, while maintaining the y sign to all patients during the operation for: A) hemorheological parameters; B) for the parameters of inflammation; C) for parameters determining anemia in the same patients. In matrix $A$ are following data $E A I_{c}-E A I_{s}$, when $E A I_{c}$ is $E A I_{s}$ in blood simples from v.Cubitalis and EAIs is EAI in v. Spleen Potrae in the columns for all patients separately (the first column is patient \# 1; the second column is patient \# 2; the third article is patient \# 3). Index "c "and "s "we used in all parameters for show, when we take blood: in V.Cubitalis or V. Portal Spleen. In matrix $\mathrm{B}$, data are presented $\mathrm{EAI}_{\mathrm{c}}-\mathrm{EAI}_{\mathrm{s}}$, etc. in columns for all patients individually (the first column is patient \# 1; the second column is patient \# 2; the third article is patient \# 3). Matrix C presents data $\mathrm{EAI}_{\mathrm{c}}-\mathrm{EAI}_{\mathrm{s}}$, ect. in columns for all patients individually (the first column is patient \# 1 ; the second column is patient \# 2; the third article is patient \# 3).

We make matrixes I, II, III. This is three matrixes.

\begin{tabular}{|c|c|c|c|c|c|c|c|c|c|c|c|c|c|c|}
\hline \multirow[t]{3}{*}{ I } & 10 & 1 & 1 & 5 & \multirow[t]{3}{*}{ II } & 22 & 0 & 22 & 0 & \multirow[t]{3}{*}{ III } & 3 & 0 & 2 & 0 \\
\hline & 15 & 1 & 1 & 5 & & 2 & 0 & 13 & 12 & & 12 & 0 & 0 & 0 \\
\hline & 8 & 1 & 1 & 12 & & 10 & 0 & 31 & 85 & & 20 & 2 & 0 & 2 \\
\hline
\end{tabular}

We calculated the ranks of the matrixes. The rank of all matrixes equals to $3 \mathrm{rd}$. Next, we laid them skeletal in $A_{I}, A_{I I}, A_{I I I}$.

This is matrix I equals $A_{\mathrm{I}}$.

\begin{tabular}{|c|c|c|c|c|c|c|c|c|c|c|c|c|c|}
\hline \multirow[t]{3}{*}{$\mathrm{A}_{\mathrm{I}}=$} & 10 & 1 & 1 & 5 & \multirow[t]{3}{*}{$\mathrm{P}=$} & 0 & 0 & 1 & \multirow[t]{3}{*}{$\mathrm{Q}=$} & 15 & 1 & 1 & 5 \\
\hline & 15 & 1 & 1 & 5 & & 1 & 0 & 0 & & 8 & 1 & 1 & 12 \\
\hline & 8 & 1 & 1 & 12 & & 0 & 1 & 0 & & 10 & 1 & 1 & 5 \\
\hline
\end{tabular}

This is matrix II equals $\mathrm{A}_{\mathrm{II}}$.

\begin{tabular}{|c|c|c|c|c|c|c|c|c|c|c|c|c|c|}
\hline \multirow{3}{*}{$\mathrm{A}_{\mathrm{II}}=$} & 22 & 0 & 22 & 0 & \multirow[t]{3}{*}{$\mathrm{P}=$} & 0 & 0 & 1 & \multirow{3}{*}{$\mathrm{Q}=$} & 15 & 1 & 1 & 5 \\
\hline & 2 & 0 & 13 & 12 & & 1 & 0 & 0 & & 8 & 1 & 1 & 12 \\
\hline & 10 & 0 & 31 & 85 & & 0 & 1 & 0 & & 10 & 1 & 1 & 5 \\
\hline
\end{tabular}

This is matrix III equals AIII.

\begin{tabular}{|c|c|c|c|c|c|c|c|c|c|c|c|c|c|}
\hline \multirow[t]{3}{*}{$\mathrm{A}_{\mathrm{III}}=$} & 3 & 0 & 2 & 0 & \multirow[t]{3}{*}{$\mathrm{P}=$} & 0 & 0 & 1 & \multirow[t]{3}{*}{$\mathrm{Q}=$} & 20 & 2 & 0 & 2 \\
\hline & 12 & 0 & 0 & 0 & & 1 & 0 & 0 & & 12 & 0 & 0 & 0 \\
\hline & 20 & 2 & 0 & 2 & & 0 & 1 & 0 & & 3 & 0 & 2 & 0 \\
\hline
\end{tabular}

The rows of the single matrix are linearly independent.

The nature of the linear dependences of the matrix does not change with a random transposition of its rows or columns. According to the existing theorems of mathematical operations on matrixes, rows of a matrix are dependent only if one of them is a linear combination of the other rows and if some of the lines are linearly dependent, then all of them are linearly dependent. Consequently, if there is a zero row among the rows of the matrix, then these rows are linearly dependent, and if there are proportional rows among the rows of the matrix, then all of them are linearly dependent. Based on this, it is clearly seen that the linear dependence of the parameters exists only in the hemorheological component of blood fluidity. We will try to explain this, relying on the physiological features of the blood and its' circulation. Blood is inhomogeneous; it circulates in the systems of micro and macro-circulation, having its own patterns. The continuity of movement is provided by the pressure gradient at the ends of the vessels and resistance, which 
is overcome by the blood flow. Under normal physiological conditions, the blood flow is characterized by comparative constancy and compensatory adequacy. However, with all pathophysiological abnormalities, this process is disturbed. This is especially noticeable in oncological diseases, when resistance to blood flow is caused not only by changes in the composition of blood and plasma, but also by mechanical barriers. From the mechanics of blood flow point of view (not to be confused with the mechanics of blood circulation), we paid attention to the set of factors that are most important for the clinic of stomach cancer. These are hemorheological factors, factors of inflammation, factors that cause an anemic condition or, on the contrary, hyperhemoglobinemia. Blood flow is ensured by two parallel systems (on the one hand, it is rheological, and on the other hand, it is coagulative-anticoagulation). The question may arise: why we did not consider the parameters of the hemostasiogram in our work (coagulogram). As for the standards proposed for the treatment of stomach cancer, as well as other operable cancer diseases, on the day of cancer diagnosis in order to prevent operative and postoperative complications, anticoagulation therapy is prescribed to patients, regardless whether the patient's coagulation system is impaired or not. We do not focus on coagulation mechanisms in this work, since we cannot identify them with reality in view of the anticoagulant therapy received by patients in accordance with accepted standards of treatment of such patients. However, we investigated the adjacent parameter (FIB), which is one of the anticoagulant factors that in the scientific literature is often identified with inflammatory processes [9]. Fibrinogen under the influence of exocoagulants is the most static of the parameters of hemostasiology, so it can be determined as a factor of inflammation. According to the laws of theoretical biomedicine, resistance and pressure difference at the ends of blood vessels act as a basis of blood circulation. The resistance to blood flow depends on the nature of the blood flow - turbulent or laminar. Under conditions of physiological rest in almost all parts of the circulatory system, laminar flow, i.e. a layered blood flow, is observed without turbulence and layer mixing. A plasma layer is located near the vessel wall, the speed of which is limited by the fixed surface of the vessel wall, and the layer of erythrocytes moves along the axis at high speed. The layers slide against each other, which creates resistance (friction) for the blood flow as a heterogeneous fluid. Shear stress arises between the layers, inhibiting the movement of the faster layer. According to our data, the parameters studied in regional networks and in the general blood circulation differed from each other. We found a particular difference in hemorheological parameters. Apparently, this is due to the diameter and angle of separation of the branch from the main artery; it may change the ratio of the volume of formed elements and plasma. This is due to the fact that in parietal blood layer there is a greater proportion of plasma, and in the axial - red blood cells, therefore, in the dichotomous division of the vessel, a smaller branch or branch that extending at a right angle receives blood with a high plasma content. The viscosity of moving blood varies depending on the nature of the blood flow and the diameter of the vessels. The length of the vessel does not have a special meaning as a factor affecting the blood flow, because we take blood directly during the operation not from the pool of the abdominal cavity, but from the leading artery. Turbulent flow is characterized by twists, while the blood moves not only parallel to the axis of the vessel, but also perpendicular. Turbulent flow is observed in the proximal sections of aorta and pulmonary trunk during the expulsion of blood from the heart, local turbulence can be created in places of branching and narrowing of the arteries, in the area of sharp bends of the arteries. The movement of blood can become turbulent in all large arteries with an increase in the volumetric blood flow velocity (for example, with intensive muscular work) or with severe anemia. Turbulent movement significantly increases the internal friction of blood, and for its advancement, much more pressure is required. Hemorheological parameters in the local blood were significantly changed compared with those in the central circulation, whereas the parameters of inflammation and determining the anemic state remained equivalent locally and centrally. This suggests that from the biomechanics point of view it is the rheological parameters that are responsible for the fluidity of the blood, which determines the condition of the patient in the postoperative period. The postoperative period determines the outcome of treatment of such patients. Rheological parameters are responsible for resistance and even for the total length of the vessels. In view of the rheology laws, smallcaliber vessels are often blinded, especially during pathological processes. Sometimes, on the contrary, angiogenesis occurs, anastomoses grow, new small vessels open and the length of the common network of blood vessels increases. But as we indicated above in our specific cases, the $\Delta$ th length of a specific vessel can be neglected, because we take blood directly from the adducting artery during the operation. The process of taking blood directly from the artery during the operation is our technical knowhow. This is the most optimal method to monitor several body systems simultaneously at the site of tumor growth $[10,11]$. Our proposed tactics do not increase the duration of the operation, the risks of complications remain unchanged. Blood circulation of the organ itself does not change due to the small size of a vessel. The obliteration of the vessel produced by us is the prevention of local bleeding, thrombosis, and stasis. It 
would be appropriate to continue research in this direction, to investigate a large cohort of patients, not only before/during the operation, but also in the postoperative period. We hope that our joint research of hemorheological, inflammatory parameters, as well as of the parameters that determine anemia in patients who suffer from a stomach cancer, will initiate multidisciplinary research. For the first time in this article, we were able, to determine the variability of blood flow in the central and local blood circulation during stomach cancer, by means of elementary mathematical transformations, to find out which system provides this change. Our findings are based on basic knowledge of biomedicine blood circulation in cancer diseases and are based on mathematical determinants.

Conclusions. You can list only a number of assumptions about the most likely stimuli and manifestations of the disease, but none of them can act separately, in each case we should speak about a complex of possible factors that have not yet been fully studied in the context of multidisciplinary biomechanics and biomedicine. In this article, for the first time, we were able to determine the variability of blood flow in the central and local blood circulation during stomach cancer, by means of elementary mathematical transformations, in order to find out which system provides this kind of change. Our findings are based on basic knowledge in the sphere such as biomedicine, blood circulation in cancer diseases and are based on mathematical determinants.

\section{REFERENCES}

1. Mantskava M., Davlianidze L., Momcelidze N. Rheological properties of Blood (experimental study). https://doi.org/10.15360/1813-9779-2014-5-27-32

2. "Gastric Cancer Treatment (PDQ®)". NCI. April 17, 2014. Archived from the original on July 5, 2014. Retrieved July 1, 2014.

3. http://rostgmu.ru/wp-content/uploads/2014/12/WMA_Helsinki.pdf

4. Korotkova E., Ivannikov A., Ognerubov N. et al. Stomach cancer: molecular biological features // Tambov University Bulletin. Series: Natural and Technical Sciences. 2014. №3. URL:https://cyberleninka.ru/article/n/rak-zheludka-molekulyarno-biologicheskie-osobennosti

5. Martynova Yu.S., Martynov A.N., Turkov V.G., Kletikova L.V. The role of proteins of the acute phase of inflammation in the pathogenesis of pyometra //Scientific notes KGAVM them

6. N.E. Bauman. 2015. №1, p.5 URL: https://cyberleninka.ru/article/n/rol-belkov-ostroy-fazy-vospaleniya-vpatogeneze-piometry

7. Ruddon R. Cancer biology (4th ed.). Oxford: Oxford University Press. p. 223. ISBN 9780195175431. Archived from the original on September 15, 2015.

8. Tyrtyshnikov E. Matrix analysis and linear algebra. - Fizmatlit, 2007-01-01. - 488 p. - ISBN 9785922107785.

9. Wagner A., Syn N., Moehler M. et al. "Cochrane Database of Systematic Reviews". The Cochrane Database of Systematic Reviews. 8: CD004064. doi: 10.1002 / 14651858.cd004064.pub4. PMID 28850174., 2017

10. World Cancer Report 2014. World Health Organization. 2014. pp. Chapter 5.4. ISBN 978-9283204299

11. World Cancer Report 2014. World Health Organization. 2014. ISBN 978-9283204299. 